\title{
La fleur, signe de grâce dans la céramique attique
}

\section{Nikolina Kei}

\section{OpenEdition}

\section{Journals}

Édition électronique

URL : http://journals.openedition.org/imagesrevues/142

DOI : 10.4000/imagesrevues.142

ISSN : 1778-3801

\section{Éditeur :}

Centre d'Histoire et Théorie des Arts, Groupe d'Anthropologie Historique de l'Occident Médiéval, Laboratoire d'Anthropologie Sociale, UMR 8210 Anthropologie et Histoire des Mondes Antiques

\section{Référence électronique}

Nikolina Kei, «La fleur, signe de grâce dans la céramique attique », Images Re-vues [En ligne], 4 | 2007, document 8, mis en ligne le 01 janvier 2007, consulté le 30 janvier 2021. URL : http://

journals.openedition.org/imagesrevues/142; DOI : https://doi.org/10.4000/imagesrevues.142

Ce document a été généré automatiquement le 30 janvier 2021.

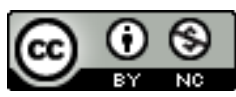

Images Re-vues est mise à disposition selon les termes de la Licence Creative Commons Attribution -

Pas d'Utilisation Commerciale 4.0 International. 


\title{
La fleur, signe de grâce dans la céramique attique
}

\author{
Nikolina Kei
}

Dans l'imagerie de la céramique attique, comme dans tous les systèmes figuratifs, certains éléments ont une valeur de signe. Postures, comportements, expressions, gesticulations mais aussi inscriptions, costumes et divers objets tenus par des figures ou meublant le champ, assument le rôle d'un idéogramme: ils renferment un message qui appelle à son décryptage.

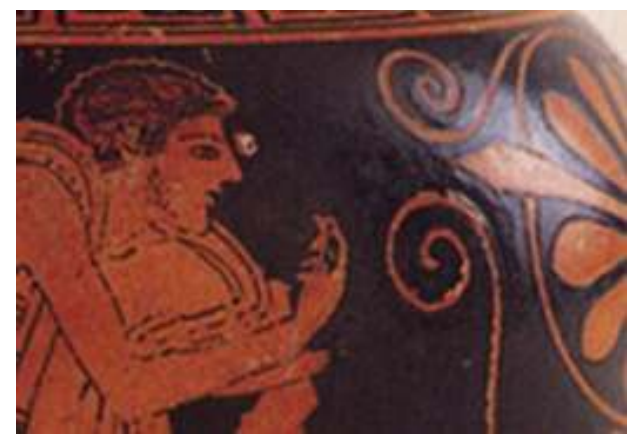

2 Parmi ces signes iconographiques qui aident à l'exégèse de l'image figurent les fleurs: sur les vases, on peut voir des personnes, mortelles ou divines, masculines ou féminines, tenir, respirer ou offrir des fleurs. Cette variété de modes de représentation investit les fleurs d'un symbolisme qui, loin d'être immuable, change selon le contexte narratif. L'interdépendance entre le signe et le cadre dans lequel ce symbolisme fait son apparition nous oblige à appuyer nos interprétations sur les séries thématiques formées par les images; ce sont elles qui nous permettent de déceler dans les schémas iconographiques, les répétitions, les variations ainsi que les exceptions ${ }^{1}$.

3 Les informations tirées de la littérature aident aussi à la compréhension d'un signe visuel. Entre l'imagerie de la céramique et la tradition littéraire grecque, il y a des affinités qui peuvent s'avérer très révélatrices du contenu symbolique, non seulement des fleurs, mais de chaque élément iconique. Nées et élaborées dans le même cadre social, les deux types d'expression se servent souvent des mêmes signes et des mêmes métaphores afin de véhiculer les concepts et les valeurs du monde grec antique. Cependant, même si la comparaison entre l'imagerie iconographique et littéraire révèle souvent une correspondance remarquable, il serait imprudent de projeter sur les images les modèles élaborés dans les textes; cette procédure négligerait le fait que 
texte et image restent toujours deux modes d'expression distincts et que toute projection systématique engendre des « sur interprétations ».

4 Les vases qui servent de base à notre analyse sont tous attiques et sont datés de la fin $\mathrm{du} \mathrm{VI}{ }^{\mathrm{e}}$ au milieu $\mathrm{du} \mathrm{V}^{\mathrm{e}}$ siècle avant notre ère. Notre visée peut être résumée ainsi : nous relèverons la fonction et les connotations diverses de la fleur dans les images et en même temps, là où cela nous paraîtra utile, nous démontrerons les parallèles entre le rôle de la fleur dans les images et dans les textes. Avant de commencer à regarder ces images, rappelons que les fleurs occupent une place privilégiée dans le monde de l'esthétique ; leurs formes, leurs coloris variés souvent éclatants, et leurs parfums, les rendent agréables aux sens. Ceci explique partiellement pourquoi le signe de la fleur a souvent été choisi afin d'embellir les images aussi bien céramiques que poétiques ; nous verrons pourtant que le rôle du signe floral n'a jamais été restreint à celui d'un simple ornement.Au médaillon d'une coupe datant des alentours de 480-470 av. J.C. ${ }^{2}$ (fig. 1), on voit une jeune femme respirer un bouton de fleur. Un lit, un miroir et une corbeille à laine, kalathos, indiquent de manière elliptique l'intérieur d'un gynécée. Tous les éléments dans l'image sont chargés de sens et articulent un ensemble iconographique cohérent en accord avec la représentation que l'on avait de la femme au Vème siècle avant notre ère.

Fig.1.

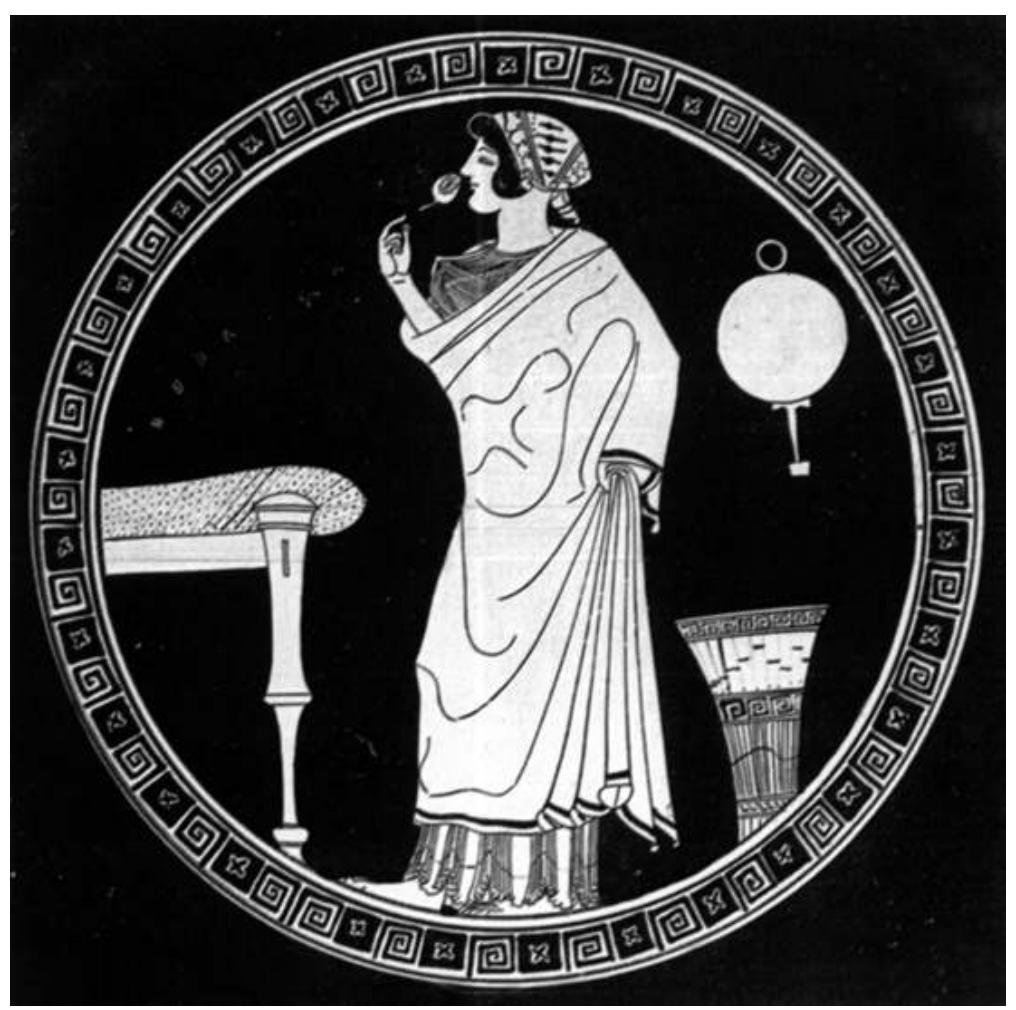

Coupe à figures rouges, à la manière de Douris. Londres, British Museum GR 1843.11-3.94 (E51) http://www.britishmuseum.org/

5 Examinons le mobilier ; le kalathos, posé au sol, est le signe de l'activité féminine à l'intérieur de l' oikos (le foyer), à savoir le travail de la laine. Il s'agit d'un objet typique qu'on offrait comme cadeau aux jeunes mariées, et qui les accompagnait durant toute leur vie ${ }^{3}$. Au-dessus du kalathos, comme pendu au mur, figure un miroir ${ }^{4}$, qui étant 
associé aux apprêts de la femme, au kosmos féminin, est porteur d'une forte signification érotique : il permet à la femme de se parer et de confirmer sa beauté afin d'exercer son charme sur les hommes. A gauche, le lit, loin d'être réduit à un simple indice spatial, fait allusion aux futures rencontres amoureuses de la jeune femme. Ainsi, tous ces objets dépassent leur aspect utilitaire dans le cadre du foyer et deviennent ici signes de la féminité.

Néanmoins, ce n'est pas seulement le mobilier féminin qui rend une valeur allusive, mais aussi la tenue de la jeune femme qui participe à l'érotisation de la scène; le manteau qui enveloppe son corps ne couvre pas son sein droit. Il y a un jeu de la part du peintre, croyons-nous, entre l' aidôs de la fille (notion qui englobe honte, pudeur, modestie, respect et honneur) matérialisée dans l'image par le large manteau ${ }^{5}$, et le désir érotique que peut provoquer la vue de son beau corps. A l'accentuation de la beauté de la jeune fille participe également la présence de la fleur. En tant qu'objet de plaisir visuel elle-même, la fleur transforme aussi la personne qui la tient en objet d'admiration; déesses, dont la beauté et l'éclat sont incomparables, jeunes filles, parthenoi à la fleur de l'âge anthos hêbès prêtes pour leur éclosion à travers le mariage ${ }^{6}$, ainsi que jeunes femmes au statut social indéfinissable apparaissent souvent dans l'imagerie attique avec une fleur à la main. Celle-ci devient une sorte de parure qui relève le charme naturel de la femme, sa charis ${ }^{7}$. La poésie mélique, qui se développe du milieu du VIIème au milieu du Vème siècle avant notre ère, vient à notre secours pour confirmer l'articulation entre le signe floral et les concepts de la grâce et de la séduction. L'image d'une belle adolescente tenant une fleur est très récurrente; on pourrait même dire qu'il se produit souvent une véritable adéquation entre la fille et la fleur. Archiloque dépeint ainsi une belle image de grâce féminine :

Elle se plaisait à tenir à la main une branche de myrte ou la belle fleur du rosier, et sa chevelure abritait en ombrelle sa nuque et ses épaules 8 .

7 L'être aimé qui inspira ces vers avec l'innocence de son âge, s'amuse dans une prairie avec des fleurs dans les mains. On est bien dans l'esthétisme floral : le rameau de myrte et la belle fleur du rosier soulignent le charme dû à sa jeunesse et rehaussent sa capacité de séduction. Sappho, elle aussi, aime chanter la beauté de jeunes filles tendres et gracieuses. Dans ses images poétiques, des fleurs et des odeurs fines et douces se mêlent et exercent leur pouvoir envoûtant sur la poétesse.

Oui, combien de couronnes de violettes, de roses et de safrans à la fois tu posais sur la tête à côté de moi ! Combien de guirlandes tressées, de charmantes fleurs, tu enlaçais autour de ta gorge délicate! Combien de vases de parfum, brenthium ou royal, tu répandais sur ta belle chevelure! Ou, couchée, près de moi, sur un lit moelleux tu apaisais ta soif ${ }^{~}$. Cependant, l'image de la fleur peut être chargée par les poètes de connotations négatives, surtout lorsqu'elle est assimilée à la féminité. La fleur est soumise aux incidences du temps comme d'ailleurs la femme ellemême ; elle s'effeuille, elle se fane.

La fleur de sa virginité s'est effeuillée et avec elle la grâce qui était la sienne auparavant ${ }^{10}$.

8 Ici la fleur de la virginité, anthos partheneion, voire l'essence virginale, flétrit et la femme perd sa beauté. Or, ce n'est pas seulement l'écoulement du temps qui anéantit la fleur mais aussi la présence de l'homme qui semble ne pas respecter sa beauté et sa fragilité. Chez Sappho, la virginité assimilée à une fleur, est toujours en jeu :

Comme la jacinthe que, dans les montagnes, les bergers foulent aux pieds- et voici que (s'effeuille) à terre sa fleur pourpre ${ }^{11}$. 
tardive mais très révélatrice sur le caractère éphémère des fleurs :

Je t'envoie Rhodocleia ${ }^{12}$, cette couronne qu'avec de belles fleurs j'ai tressée de mes propres mains. Il y a des lis, des boutons de rose, des anémones humides, des narcisses flexibles, des violettes aux sombres reflets. Mets-la sur la tête et cesse d'être si fière : vous fleurissez et vous passez, toi comme la couronne (antheis kai klègeis kai su kai ho stephanos $)^{13}$.

10 Les fleurs, que ce soit dans la poésie ou dans la céramique, rappellent que la jeunesse et le charme sont tous les deux précaires et que les plaisirs qu'ils engendrent sont fugaces ${ }^{14}$. Revenons à notre image. La fleur ne se restreint pas à résonner la beauté juvénile, pourtant éphémère, elle évoque également le parfum du corps de la fille, arme féminine indispensable dans les stratégies de séduction ${ }^{15}$. Ceci ne doit pas nous surprendre car les fleurs constituent la matière première pour la confection des arômes et des huiles auxquels les Grecs accordaient des vertus aphrodisiaques. Dans l'Iliade, la fameuse scène de séduction de Zeus par sa femme Héra démontre que le parfum fait partie des artifices qui suscitent le désir des hommes tout comme les jolis vêtements, les bijoux et nombre d'autres ornements :

Avec de l'ambroisie ${ }^{16}$ elle efface d'abord de son corps désirable toutes les souillures. Elle l'oint ensuite avec une huile grasse, divine et suave, (lip'elaiô ambrosiô edanô) dont le parfum est fait pour elle ${ }^{17}$.

11 Les apprêts d'une autre déesse, Aphrodite, sont marqués par toute une série de sensations olfactives :

Elle allait à Paphos, l'Aphrodite aux sourires retrouver son enclos, l'encens de son autel (bômos thyeis), et, l'ayant mise au bain, les Grâces la frottaient de cette huile divine (elaiô ambrotô) qui reluit sur la peau des dieux toujours vivants, puis elles lui passaient une robe charmante, enchantement des yeux $!^{18}$.

12 L'univers de la déesse est imprégné des senteurs enivrantes qui émanent de son temple, de son autel, de l'huile immortelle avec laquelle les Grâces, Charites, frottent son corps, et enfin de la Chypre entière, l'île de tous les parfums dans la conception des Grecs. A l'instar d'Aphrodite, toutes les femmes doivent sentir bon. Au cas contraire, elles risquent de connaître le sort des Lemniennes de la légende quittées par leurs maris parce qu'émanait d'elles une odeur repoussante ; c'est ainsi qu'Aphrodite les a punies pour avoir négligé de l'honorer correctement ${ }^{19}$. La déesse qui préside aux rencontres amoureuses a ses fleurs chéries, la rose et le myrte, avec lesquels les femmes tressaient des couronnes en son honneur. On considérait comme un bon présage pour une jeune fille de confectionner elle-même des couronnes et de les donner à son futur mari le jour de leur mariage $e^{20}$. Les jeunes époux parés des fleurs et aspergés de parfums étaient au comble de leur charis, qui est le fondement du lien conjugal. Mais les parfums, les onguents et les huiles trouvent leur place également en dehors du cadre matrimonial ; qu'il s'agisse d'une jeune épouse ou de n'importe quelle autre femme, elles ont toutes recours à la parfumerie afin de rehausser leur pouvoir attractif. Le lien des fleurs aux arômes est manifeste sur la coupe du British Museum. Si on regarde à l'extérieur du vase, on voit une série de rencontres amoureuses entre hommes et femmes (fig. 2) ${ }^{21}$ : deux des quatre femmes tiennent des alabastres, à savoir des vases à parfums qui renvoient tout comme le bouton du médaillon à l'univers des fragrances. Ainsi, le peintre choisit deux objets, la fleur et l'alabastre, afin de rendre visible ce qui ne peut pas être illustré : le parfum, dont le rôle dans le jeu de la séduction féminine est primordial. La jeune fille accumule ainsi toutes les qualités qu'une femme devait avoir 
selon les concepts de la société athénienne : elle est ardente au travail (philergos) et belle à voir. Même si sur le médaillon de notre vase nous ne voyons pas de représentation d'hommes, rappelons que la coupe était utilisée dans le cadre du banquet et qu'une jolie jeune fille tenant une fleur aurait suscité l'admiration des spectateurs, à savoir les convives du symposion.

Fig.2.

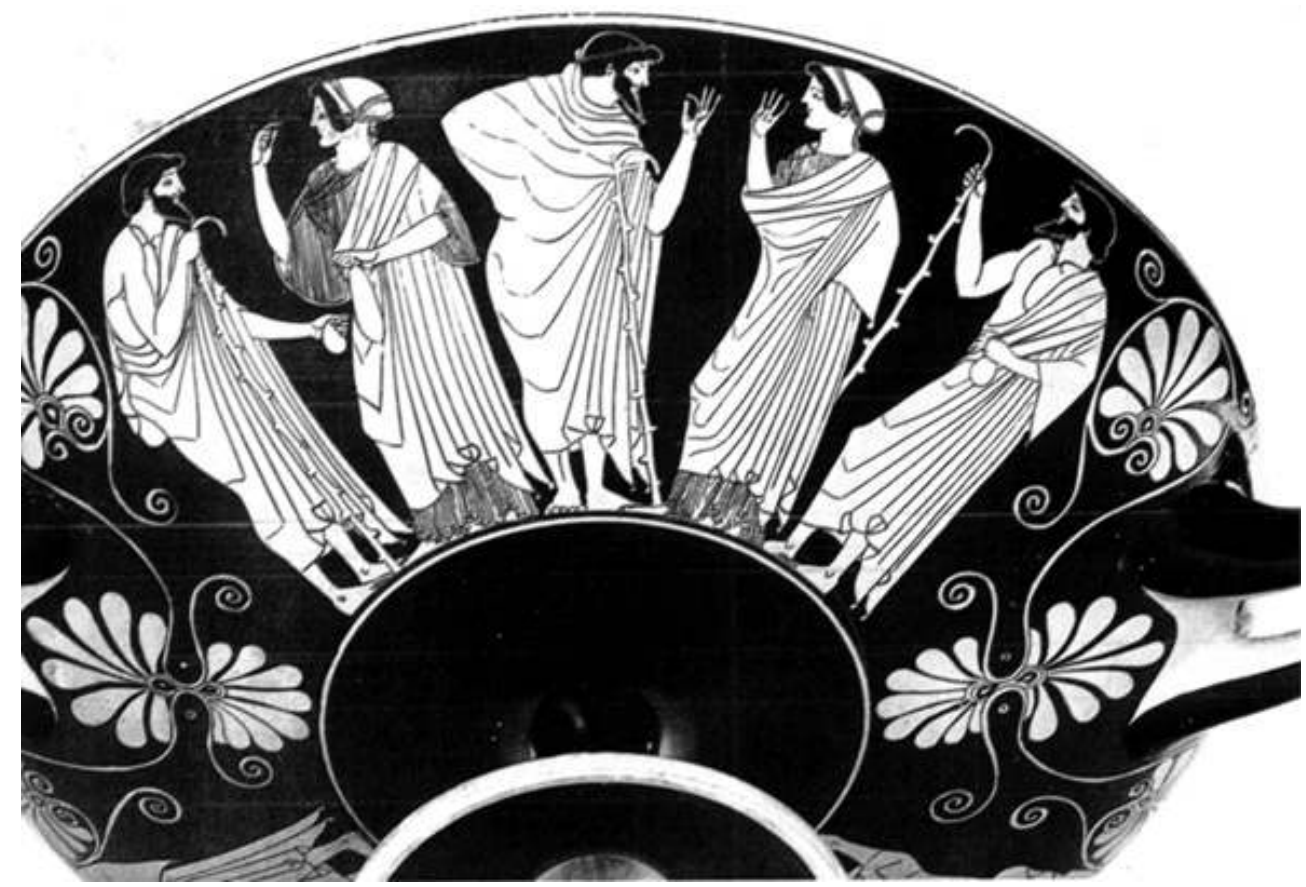

Coupe à figures rouges, à la manière de Douris. Londres, British Museum GR 1843.11-3.94 (E51)

http://www.britishmuseum.org/ 
Fig.3.

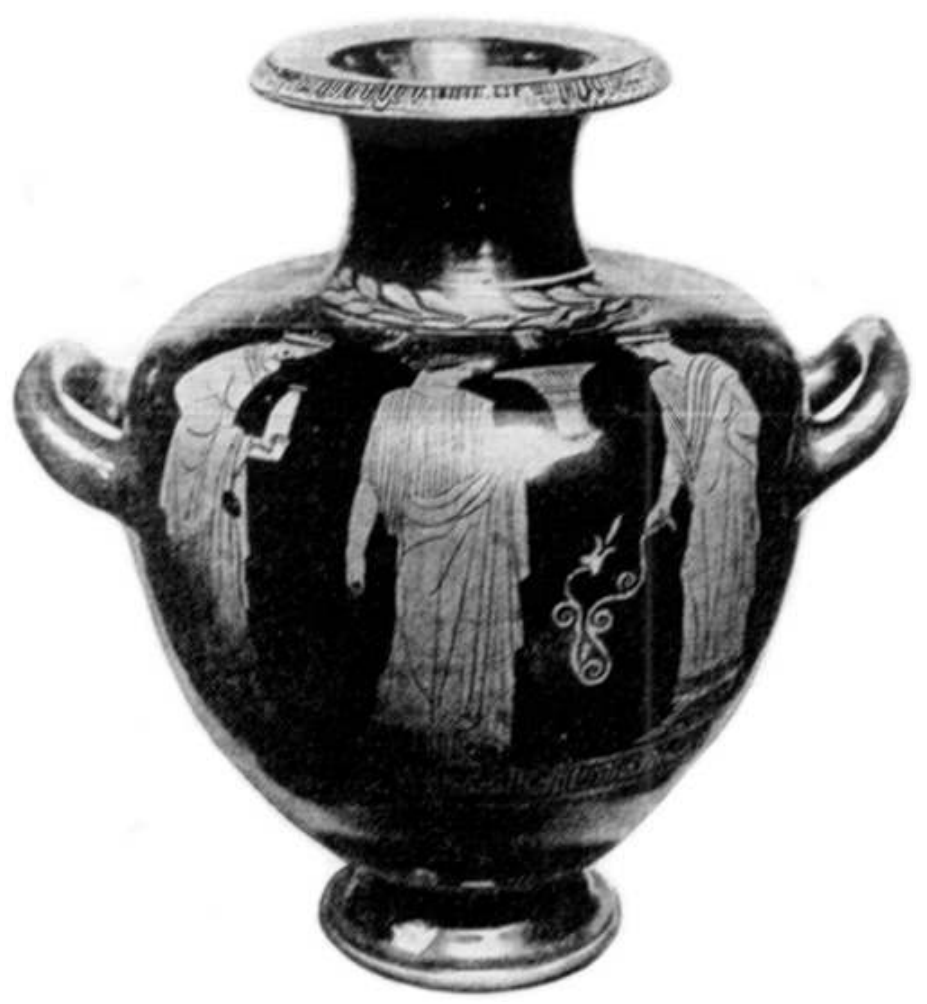

Hydrie à figures rouges, Peintre de la Centauromachie du Louvre. St Petersburg, Musée de l'Hermitage 4525

http://www.hermitagemuseum.org/

La même remarque vaut pour une hydrie de St Petersburg ${ }^{22}$ (fig. 3), où on voit trois jeunes femmes tenant des objets : la première à gauche tient un alabastre, celle au centre une corbeille à laine et la troisième à droite tient un rameau fleuri. G. Ferrari ${ }^{23}$ suggère que cette image n'est pas complète en elle-même et que les figures renvoient à un contexte plus large, celui de la toilette féminine dans lequel ces femmes joueraient le rôle d'assistantes. En effet, ces trois objets articulent un nexus de valeurs associé au registre du kosmos féminin. D'une part, le grand rameau fleuri referme graphiquement le contenu de l'alabastre, à savoir le parfum, et d'autre part, il est comme un ornement qui met en avant la grâce de sa silhouette. Deux éléments accentuent son caractère symbolique: d'une part son graphisme extrêmement stylisé, semblable à celui des motifs floraux des zones secondaires des vases, et d'autre part le fait que la femme ne le tienne pas de façon à le respirer mais à la façon d'une bandelette. On comprend alors que par le biais de trois objets assemblés dans la même image, le travail de la laine s'associe au registre de la parure afin de construire le concept de la femme athénienne idéale. 
Fig.4.

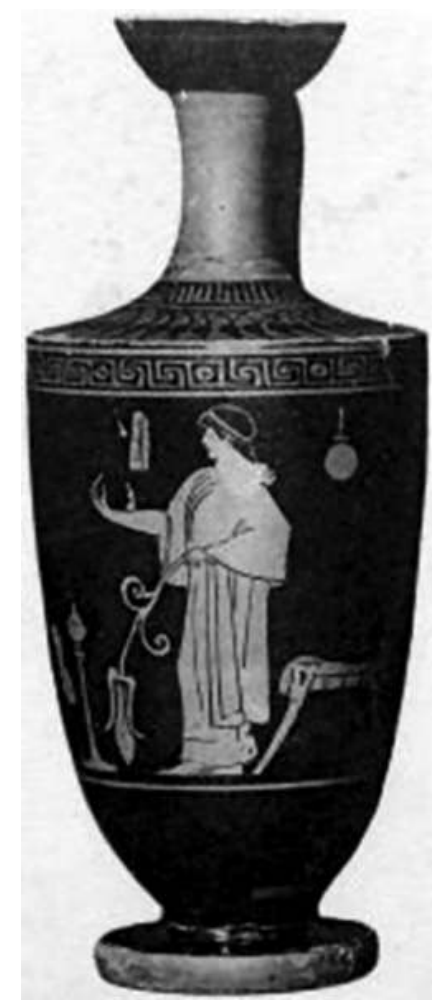

Lécythe à figures rouges, Peintre de Dutruit. New York, Metropolitan Museum of Art 41,162.27 http://www.metmuseum.org/

Passons à un autre exemple, celui d'un lécythe à figures rouges attribué au Peintre de Dutuit et datée de 470 av. J.C. ${ }^{24}$ (fig. 4). Une jeune femme est figurée debout à l'intérieur d'un gynécée, indiqué par une chaise, un miroir, une bandelette et un encensoir, thymiatérion ${ }^{25}$. Elle tient deux fleurs : l'une, dans sa main droite, colorée en rouge, est à peine visible et évoque une vraie fleur, tandis que l'autre est étonnamment grande et son graphisme rappelle celui des ornements floraux des vases. Pour quelle raison le peintre a-t-il choisi de mettre dans les mains de la figure deux fleurs aussi différentes ? S'agit-il d'une manière d'attirer l'attention du spectateur du vase? On remarque pourtant que même si la fleur ornementale s'impose dans l'image avec son volume, la femme met en avant avec son geste la petite fleur presque invisible. De cette manière, un équilibre s'établit entre les deux qui acquièrent une importance égale dans l'image. Tentons d'interpréter le choix du peintre d'illustrer ces deux fleurs si différentes. Il est possible que le rôle de la fleur "mimétique " soit d'indiquer la beauté juvénile de la fille, sa grâce et peut-être son parfum. L'image orne un lécythe, vase destiné à la conservation des huiles parfumées; ainsi il est légitime de dire que le contenu de la représentation renvoie au contenu de son support. La fleur stylisée, à proximité de l'encensoir, pourrait matérialiser graphiquement les fumigations des aromates brûlés. En tout cas, nous avons ici une image " odorante " qui laisse le spectateur imaginer le parfum de la jeune fille, la senteur des fleurs et les fumeroles des aromates; image idéale pour un vase à parfum.

La fleur occupe très fréquemment, dès la fin de l'époque archaïque, une place centrale dans la schématisation iconographique des couples (homo ou hétérosexuels) appartenant aux scènes d'échanges amoureux ${ }^{26}$. Celles-ci sont l'illustration de la 
stratégie érotique selon laquelle un homme attire l'objet de son désir, qu'il s'agisse d'un adolescent ou d'une jeune femme, avec un cadeau. On a souvent interprété ce geste comme une manière d'engager l'autre dans une relation érotique, philotès, lecture qui assimilait les femmes figurées à des prostituées. Même si c'est souvent le cas, l'imagerie d'échange de dons se caractérise par une telle variation de gestes, d'attitudes, de contextes spatiaux et d'autres détails significatifs que ce serait une erreur de se restreindre à une seule grille interprétative. L'acte d'offrir un cadeau pourrait impliquer un assez grand degré d'affection tout en établissant entre les deux personnes un certain rapport, pas forcément charnel.

Fig.5.

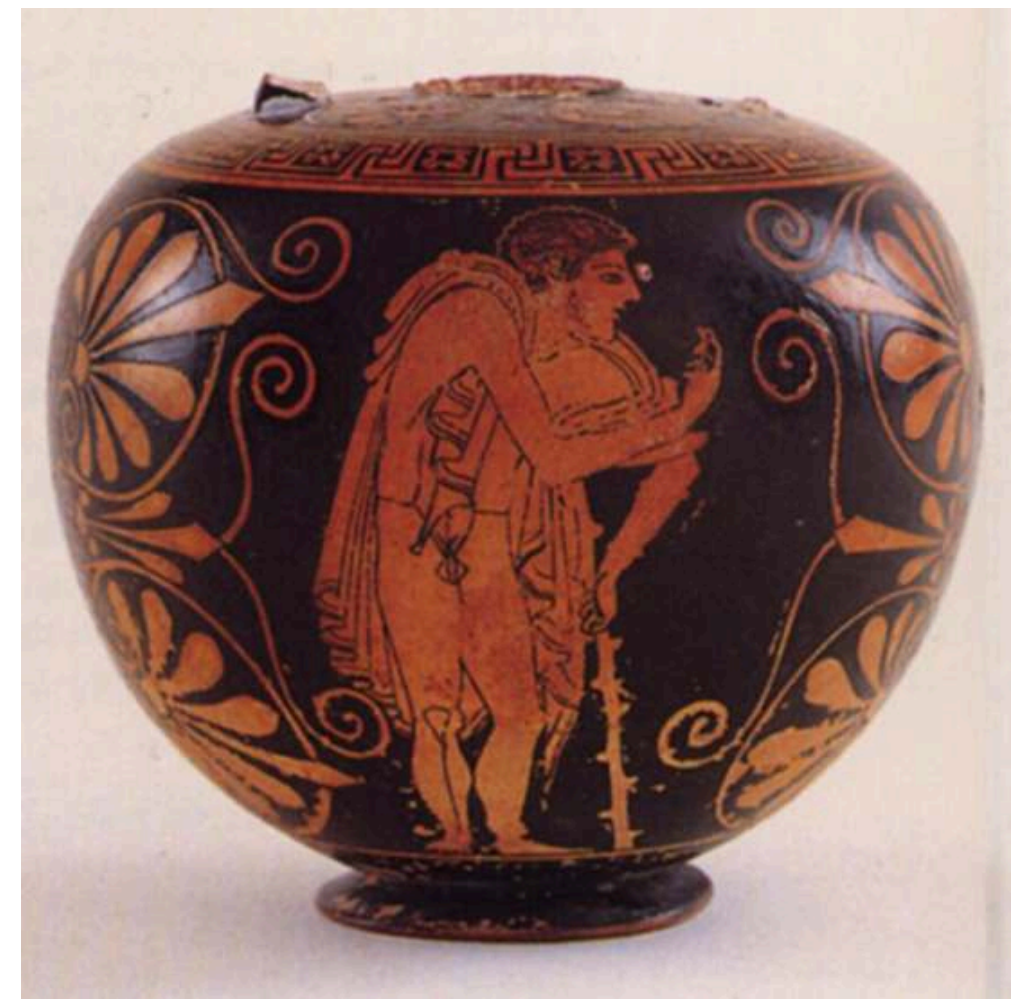

Aryballe à figures rouges, Douris. Athènes, Musée National, Troisième Ephorie 556 http://odysseus.culture.gr/h/1/eh154.jsp?obj_id=3249 
Fig.6.

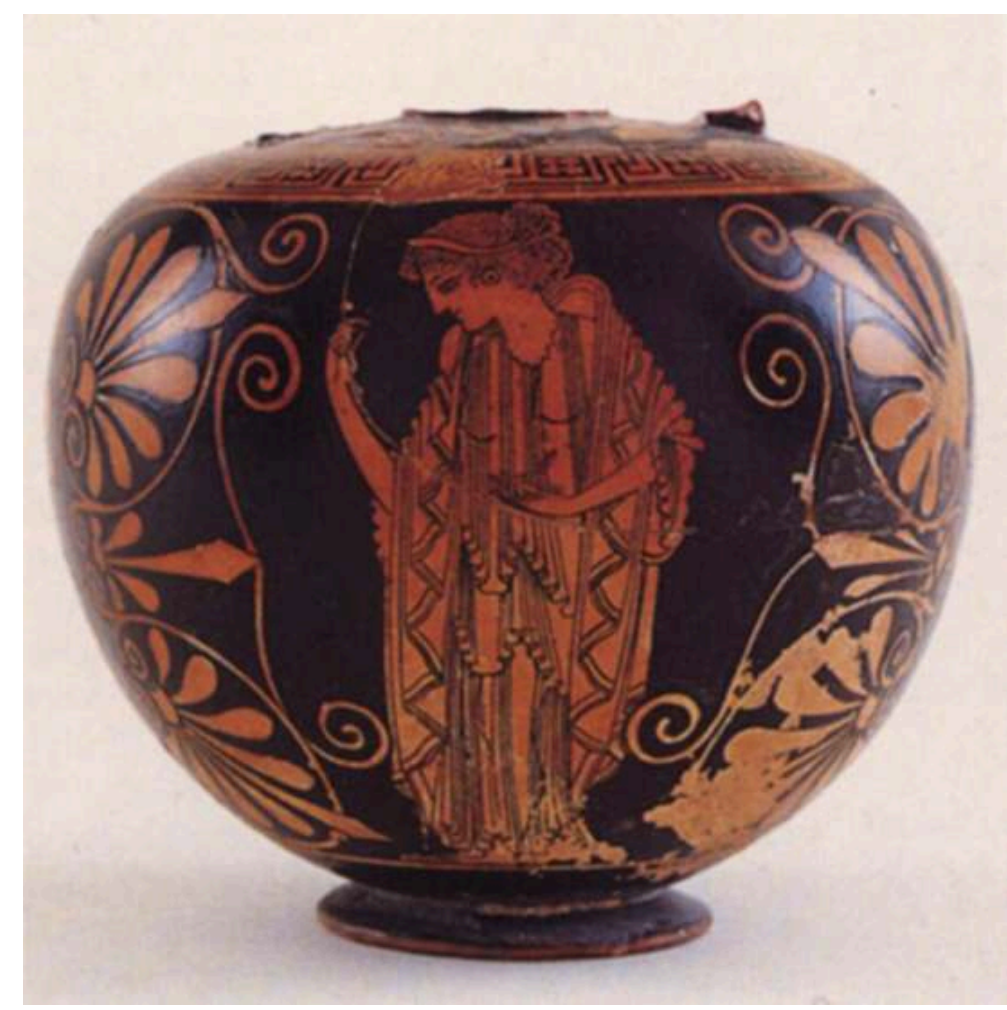

Aryballe à figures rouges, Douris. Athènes, Musée National, Troisième Ephorie 556 http://odysseus.culture.gr/h/1/eh154.jsp?obj_id=3249

16 Un aryballe à figures rouges de Douris $^{27}$ qui date de 510-505 av. J.C. (fig.5 et 6), représente d'un côté une jeune fille et de l'autre un jeune homme s'appuyant sur son bâton. Bien que les deux figures soient séparées d'une riche décoration végétale, elles font partie de la même scène. Le jeune homme tend une fleur à la jeune fille charmante qui a la tête légèrement inclinée et les yeux baissés, ce qui n'est pas sans importance, car l' aidôs d'une personne se manifeste dans le regard ${ }^{28}$. La fille " timide ", suivant les règles du bon comportement que les jeunes athéniennes devaient avoir à l'égard des hommes, évite de regarder le jeune homme dans les yeux et préfère contempler l'objet, aujourd'hui invisible ${ }^{29}$, qu'elle tient dans sa main droite. Heureusement, nous pouvons encore distinguer ce qu'elle tient dans l'autre main, une jolie fleur. Ici, les deux fleurs tenues par les deux personnes font référence à deux interprétations différentes de leur contenu symbolique. Pour la jeune fille, la fleur renvoie à sa jeunesse et met en avant son attrait physique, sa charis. La fleur tenue par le jeune homme assume une toute autre fonction : celle du don. Grâce à son aspect charmant et à son parfum qui stimule les sens, la fleur devient un don approprié pour courtiser les femmes.

17 La tradition littéraire en fait preuve: Athénée (Les Deipnosophistes, XII 553-554c) cite Cléarque de Soles qui dans «Erôtika» se demande pourquoi certains hommes circulent en tenant des fleurs ou des pommes dans les mains. Une première réponse pourrait être, selon lui, que les hommes s'en servent afin d'accoster la personne qu'ils désirent ; la fleur ou le fruit devient le signe de leur désir. Ces beaux cadeaux, pourtant, ne sont pas offerts gratuitement car ils exigent en échange la beauté du corps de celui qui les reçoit «è gar tôn hôraiôn anthôn kai karpôn aithèsis eis antidosin tès tou sômatos hôras prokaleitai tous lavontas $»^{30}$. Une autre réponse que Cléarque envisage, c'est que les 
hommes gardent ces objets pour eux-mêmes; leur beauté les divertit du désir de la personne aimée. Enfin, une troisième réponse serait que les personnes portent les fleurs comme des ornements personnels "peri autous kosmou» pour rehausser leur charme tout comme le font ceux qui se couronnent de fleurs. Ce petit passage démontre que les fleurs expriment avant tout un désir de beauté. De la même manière les fleurs de l'aryballe sont là pour évoquer la beauté juvénile, la séduction mais aussi le parfum conservé dans le vase.

Il existe une petite série d'images représentant des fleurs dans un contexte cultuel dénoté par la présence d'un autel. Offrandes et objets sacrificiels, les fleurs et autres substances végétales, brutes ou transformées en aromates, révèlent la conception anthropomorphique de l'approche des dieux chez les Grecs anciens ; on les déposait sur l'autel afin de vénérer les dieux ou implorer leur secours. En conséquence, on pourrait conclure que les fleurs ainsi que les essences végétales parfumées, étaient considérées comme dons de valeur, capables d'assurer la communication entre deux mondes bien séparés l'un de l'autre : le monde humain et le monde divin. D'ailleurs, le mot "parfum" (" per fumum » en latin, à savoir « à travers la fumée ») comporte un sens de communion à travers l'acte cultuel.

Fig.7.

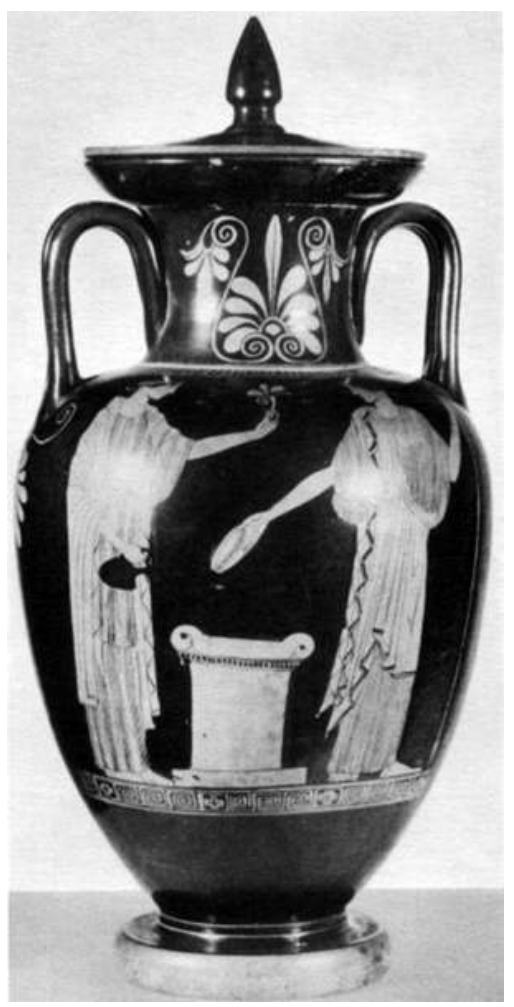

Amphore à figures rouges, à la manière du Peintre d'Altamura. Vienne, Kunsthistorisches Museum IV 772

http://www.khm.at/

19 Une amphore de Vienne ${ }^{31}$ (fig.7) représente devant un autel une jeune fille tenant dans la main gauche une oenochoé et dans l'autre main une fleur en plein épanouissement. De l'autre côté de l'autel, on voit la divinité elle-même (Héra ?) avec son sceptre et son polos. Ici, donc, l'élément floral associé à l'autel est revêtu d'une dimension religieuse 
tout comme l'oenochoé ; c'est une offrande en l'honneur de la déesse, autrement dit un signe de charis, qui cette fois désigne non pas la grâce physique, mais cet échange des dons offerts de la part des hommes et des bienfaits compensatoires de la part des dieux. Mais le rôle de la fleur ne s'arrête pas là car elle matérialise graphiquement les fumeroles d'encens destiné à être brûlé sur l'autel. Nous pourrions aller encore plus loin et suggérer que dans l'image, la fleur devient le signe du bon parfum, euôdia, qui est le trait spécifique de la condition surnaturelle des dieux: tout d'abord car tout ce qui appartient aux dieux de l'olympe, autel, temple, vêtements, exhale des parfums, et ensuite parce que la présence divine se manifeste par une odeur merveilleuse ${ }^{32}$. Nous avons vu plus haut qu'Héra et Aphrodite aiment s'oindre d'huiles ambrosiennes. Dans l'Hymne homérique à Déméter (277-278), des effluves flottent tout autour de la déesse, et un parfum délicieux s'exhale de ses voiles "odmè d'imeroessa thyeèntôn apo peplôn skidnato ». En outre, c'est un parfum divin qui annonce à Prométhée l'arrivée des Océanides $^{33}$, de la même manière qu'Hippolyte mourant ressent la venue d'Artémis grâce à son parfum divin « theion odmès pneuma » ${ }^{34}$.

La tradition littéraire nous a laissé plusieurs exemples d'" endroits " imprégnés par des émanations divines: les prés fleuris où ont lieu les rencontres amoureuses des dieux ${ }^{35}$, les jardins d'Aphrodite ${ }^{36}$, Délos au moment de la naissance d'Apollon ${ }^{37}$, ainsi que la caverne sur l'Ida où Hermès enfant est caché ${ }^{38}$, sont tous des lieux remplis d'odeurs délectables qui marquent la présence divine. Ainsi, nous pourrions suggérer que la fleur illustrée dans un contexte cultuel est une sorte de rappel que le monde divin est odoriférant, « euôdès ».

21 En guise de conclusion, remarquons que l'objet-signe implique un déplacement du concret et du sensible à l'abstrait et au conceptuel : dans les images de l'univers féminin, par exemple, nous passons de l'illustration de la corbeille à la notion de philergia, de l'illustration du miroir, du vase à parfum et de la fleur, au registre de la parure, de la beauté et de la désirabilité.

Nous avons examiné le rôle des fleurs dans le monde des femmes, dans le contexte érotique et cultuel et nous avons constaté que c'est la polysémie qui le caractérise. Elément de modulation, le signe floral est à la fois lié à la jeunesse, au parfum, à la séduction et à la charis dans sa double dimension, celle de la grâce physique et celle de la grâce rendue dans l'échange.

23 Cependant, le contenu symbolique de la fleur n'est pas quelque chose de donné : il doit toujours être examiné par rapport aux autres objets-signes représentés dans l'image puisque c'est le système entier d'objets qui construit le sens de la narration iconique. L'élément floral, s'il est par exemple associé à un autel, ne se réfère plus au kosmos féminin, mais aux pratiques religieuses des Grecs et à leur conception du divin.

Fonction et les connotations d'une fleur dans une image doivent être, bien sûr, considérées par rapport à l'usage de l'ustensile céramique : une scène représentée sur un vase utilisé dans le cadre du banquet et une scène représentée sur un vase destiné à la toilette féminine ne s'adressent pas aux mêmes destinataires et les messages ainsi véhiculés ne sont pas toujours identiques. Dernière remarque, même si la représentation des fleurs dans la céramique est plus qu'abondante, elles sont rarement l'unique sujet des images : dans la céramique attique, il n'existe ni " natures mortes " ni pots de fleurs, tels qu'on les rencontre dans la peinture occidentale. L'élément floral, signe en système, est presque toujours associé à l'homme et à sa sphère. 


\section{NOTES}

1. Lissarrague, L'autre guerrier. Archers, peltastes, cavaliers dans l'imagerie attique, Paris-Rome, 1990, p. 10.

2. Coupe à figures rouges ; Londres, British Museum GR 1843.11-3.94 (E51); à la manière de Douris ; ARV² 449,4, 1653 ; Para 376 ; CVA Grande Bretagne 17 London 9, pl. 37a-b, 38a-b.

3. F. Lissarrague, "Women, boxes, containers : some signs and metaphors" dans E. D. Reeder (éd). Pandora, New Jersey 1995, pp. 91-101.

4. Sur le symbolisme du miroir voir F. Frontisi , "L'oeil et le miroir" dans J.P Vernant et F. Frontisi-Ducroux, Dans l'oeil du miroir, Paris 1997, pp. 51-250.

5. G. Ferrari dans son ouvrage, Figures of speech. Men and Maidens in Ancient Greece, Chicago et Londres 2002, démontre que le himation enveloppant garçons, filles et femmes constitue la métaphore picturale qui rend visible la notion d' aidos .

6. La formule antos hêbès qu'on rencontre souvent dans la tradition littéraire, parfois légèrement modifiée, renferme l'idée de la vigueur et de la grâce juvénile concernant autant les garçons que les filles au stade pré-nuptial. De la même manière, le qualificatif hôraios, désigne les jeunes et les filles dans la fleur de l'âge ; il dérive du mot hôra, à savoir " la belle saison " ou " le moment propice " pour un événement tel que l'âge du mariage. La charis et la hôra marquent ce moment où la jeune personne doit s'initier à l'acte érotique. Plutarque explique que la fille sans grâce, acharis, chantée par Sappho, (frg.49) est une fille qui n'a pas encore atteint l'âge de mariage ; Plutarque, Dialogue sur l'amour, 751d.

7. Le mot se rapporte initialement à tout ce qui engendre du plaisir, de l'agrément; E. Benveniste, Le vocabulaire des institutions indo-européennes, t. 1, Paris 1969, p. 201. Chez Homère il se rapporte à la grâce d'une personne (la beauté du visage, du corps, de la posture et du comportement), ou d'un objet (beauté de forme, éclat) et à leur pouvoir de séduction. Sur la notion de charis qui englobe également d'autres notions telles que " faveur ", " bienveillance " de la personne qui accorde et " reconnaissance ", "gratitude " de la personne qui reçoit, voir B. Maclachlan, The age of Grace ; Charis in early Greek poetry, New Jersey 1993 ; D. Saintillan, " Les "Grâces" des Grecs et la philosophie : de Platon à Hegel " dans Les Études Philosophiques, n. 4 , Paris 2003 ; V. Azoulay, Xénophon et les Grâces du pouvoir ; de la charis au charisme, Paris, 2004 (avec une bibliographie riche).

8. Archiloque, fr.40, F. Lasserre, traduction A. Bonnard, Paris,1958.

9. Sappho fr.93 , 13sq, traduction Th. Reinach, Paris 1937 .

10. Archiloque, fr.196,17-19, West.

11. Sappho, fr.113, traduction Th. Reinach, Paris 1937. Cette notion d'affinité inhérente entre corps humain et végétal est souvent attestée dans le langage grec par quelques ambiguïtés terminologiques : les mots indiquant accroissement, bourgeonnement, floraison ou déchéance physique peuvent concerner les hommes ainsi que les végétaux, tout comme en revanche les termes adaptés au corps humain peuvent valoir pour les plantes.

12. Le choix de ce nom n'est pas dû au hasard car Rhodocleia est celle qui a la réputation glorificatrice (kleos) d'une rose (rhodon), elle est aussi belle et fraîche que cette fleur.

13. Traduction P. Waltz, Paris 1960.

14. Il est intéressant de rappeler ici les trois statues acrolithes des Charites sur l'agora d'Elis, décrites par Pausanias dans sa Description de la Grèce (VI, 24, 6-7) : l'une porte une rose, la deuxième une branche de myrte et la troisième un astragale. Ici, encore la grâce incarnée par les trois déesses est combinée aux fleurs d'Aphrodite et à la jeunesse signalée par l'astragale (osselet qui servait de jouet). 
15. Sur la place des parfums et des aromates dans l'imaginaire des Anciens voir P. Faure, Parfums et Aromates de l'Antiquité, Paris, 1987 ; P. Badinou, La laine et le parfum : épinetra et alabastres: formes, iconographie et fonction, Leuven, 2003 et An. le Guérer, Le parfum : des origines à nos jours, Paris 2003.

16. Le mot ambroisie a chez Homère deux sens assez différents. D'un côté, il se rapporte à un aliment solide qui constitue la nourriture des dieux; d'un autre, il désigne un onguent parfumé destiné aux soins de beauté mais qui peut aussi servir à embaumer un cadavre humain, comme celui d'Hector ou de Patrocle.

17. Homère, Iliade XIV 170-174, traduction P. Mazon, Paris, 1956.

18. Homère, Odyssée VIII.362-6, traduction V. Bérard, Paris, 1992. Une description de ses soins de beauté presque pareille à celle de l'Odyssée, existe dans l'Hymne pseudo- homérique , lorsqu'Aphrodite se prépare à séduire Anchise ; Hymne homérique à Aphrodite, 58-66.

19. Appolodore, Bibliothèque $\mathrm{I}, 9,17$.

20. R. F. Jr. Sutton, The Interaction between Men and Women Portrayed on Attic Red-figure Pottery, Chappel Hill 1981, p. 209 et 311ss; Plutarque, Vie de Marcellus 22 ; Pline, Histoire Naturelle 15,122. Le parèdre d'Aphrodite, Éros, est aussi associé au monde des fleurs et des parfums; Théognis dans le frg . 1275-8 West, nous offre une belle image d'Éros qui se lève au moment où la terre de Chypre se couvre de fleurs tandis qu'Alcman dans le frg 58 Page représente Éros comme un enfant farouche qui s'amuse à s'abattre sur les têtes des fleurs du souchet. Dans le Banquet de Platon, 196a-b, on apprend que le dieu aime se reposer dans un lieu riche en fleurs et parfums : euanthès te kai euôdès topos.

21. Rien dans l'image n'indique la place que ces femmes occupent dans la société athénienne. Le fait qu'elles sont courtisées par des hommes ne les rend pas automatiquement hétaïres; les images ne donnent pas à voir ce qui est réel, le hinc et le nunc d'Athènes du VI ème et Vème siècles av. J.C. mais ce qui est concevable. Or, il nous semble que M. Beard a raison lorsqu'elle suggère que l'ambiguïé du statut social des femmes est délibérée de la part des imagiers attiques et que l'interprétation s'appuie sur le spectateur; M. Beard, "Adopting an approach II" in T. Rasmussen et N. Spivey (éd), Looking at Greek Vases, Cambridge 1991, p. 26-30.

22. St Petersburg, Musée d'Hermitage 4525; ARV ${ }^{2}$ 1094,99; Para 517 ; A. A. Peredol'skaia, Krasnofigurnye attischeskie vazy v Ermitazhe , Leningrad, 1967, pl. 133,1. Le vase est attribué au Peintre de la Centauromachie du Louvre et daté vers 450-440 av. J.C.

23. G. Ferrari, op . cit ., p. 33.

24. New York, Metropolitan Museum of Art 41,162.27; ARV 308, 21; CVA USA 1 New York, Hoppin and Gallatin Collection ; Gallatin Collection pl. 18, 1.

25. Sur les encensoirs en général, voir C. Zaccagnino, Il thymiaterion nel mondo greco. Analisi delle fonti, tipologia, impieghi , Rome, 1998.

26. Le motif d'échanges érotiques apparaît pour la première fois dans les scènes homoérotiques de l'imagerie à figures noires de la fin du VIème siècle et disparaît vers $430 \mathrm{av}$. J.C.

27. Athènes, Musée National, Troisième Ephorie 556; Para 376,273bis ; Add ${ }^{2} 241$; D. BuitronOliver, Douris. A Master-Painter of Athenian Red-figure Vases, Mainz/Rhein, 1995, pl. 8, n. 13.

28. Dans les Deipnosophistes, Athénée de Naucratis (XIII, 564 b) cite Aristote, d'après qui aidos réside dans les yeux : tous ophtalmos, en ois tèn aidô katoikein.Voir aussi Aristote, Rhétorique II. 6.18; Sappho frg . 23 ; Hymne homérique à Déméter, 213-215. Sur la connection de l' aidos avec la charis voir B. Maclachlan, op . cit ., p. 32-33.

29. La fille fait le même geste que l'homme ; peut-être offrait-elle, elle aussi, une fleur au jeune homme.

30. Ici, nous devons être prudents; la suggestion de Cléarque que les dons ont une finalité concrète ne fait pas une règle. Les images nous permettent d'envisager plusieurs interprétations, surtout dans le cas où la personne qui offre un don est une femme. Sur un alabastre à fond blanc attribué au Peintre de Copenhague 3830, (Palerme, Mormino Collection 796, ARV² 723,1) une 
femme présente un coq à un jeune homme, cadeau qu'on rencontre très souvent dans les scènes dites homoérotiques. Sur le sujet, voir A. Schnapp, Le chasseur et la cité : chasse et érotique en Grèce ancienne, Paris, 1997.

31. Vienne, Kunsthistorisches Museum IV 772 ; à la manière du Peintre d'Altamura ; vers 460-450 av. J.C. ; ARV 297 ; CVA Autriche 2 Wien 2, pl. 62.2.

32. Sur le sujet, voir W. Deonna, "Euodia. Croyances antiques et modernes : l'odeur suave des dieux et des élus ", Genava, t. XVII, 1939, pp. 167-262.

33. Eschyle, Prométhée enchaîné, 115sq.

34. Euripide, Hippolyte, 1391.

35. Zeus et Héra couchent sur un pré émaillé des mélilots, safrans et jacinthes (Homère, Iliade XIV 312sq); dans un pré fleuri imprégné des parfums envoûtants couchent également Poséidon et Méduse (Hésiode, Théogonie 276sq). Sur la fonction symbolique des prés et des jardins légendaires, voir C. Calame, L'Éros dans la Grèce antique, Paris, 1996, p.173-197.

36. Hymne homérique à Aphrodite 58sq et 155sq ; Homère, Iliade II 819sq.

37. Théognis, frg $.8 \mathrm{sq}$ : : " pasa men eplesthè Délos apeiresiè odmès ambrosiès ".

38. Hymne homérique à Hermès, 231.

\section{RÉSUMÉS}

Dans l'imagerie de la céramique attique, on rencontre souvent des figures, mortelles ou divines, masculines ou féminines qui tiennent, respirent ou offrent des fleurs. Ces dernières ne sont pas de simples ornements dépourvus de sens. Elles sont des signes investis d'une charge sémantique qui, loin d'être immuable, change selon le contexte narratif de l'image. Elément, donc, de modulation, le signe floral matérialise la notion grecque de la charis dans sa double dimension, celle de la grâce physique d'une personne (beauté, jeunesse, désirabilité) et celle de la grâce rendue dans un échange de toute sorte (érotique ou cultuel). Or, souvent la fleur revêt une dimension supplémentaire : elle est un signe odorant qui communique au spectateur de l'image l'impression olfactive d'un objet, d'une personne, d'un lieu ou d'une situation.

\section{INDEX}

Mots-clés : femme, fleur, grâce, homme, ornement, parfum

Index géographique : Athènes

Index chronologique : Antiquité

Thèmes : céramique

\section{AUTEUR}

\section{NIKOLINA KEI}

Nikolina Kéi est diplômée en archéologie et en histoire de l'art de l'Université Nationale

"Kapodistrias" d'Athènes, Nikolina Kéi est aujourd'hui doctorante à l'EHESS sous la direction de 
F. Lissarrage. Elle travaille autour du symbolisme des fleurs dans la céramique attique. nikolinakay665@hotmail.com 